\title{
Comments on field equivalence principles
}

\section{Appel-Hansen, Jørgen}

\section{Published in:}

I E E E Transactions on Antennas and Propagation

Publication date:

1987

\section{Document Version}

Publisher's PDF, also known as Version of record

Link back to DTU Orbit

Citation (APA):

Appel-Hansen, J. (1987). Comments on field equivalence principles. I E E E Transactions on Antennas and Propagation, 35(2), 242-244.

\section{General rights}

Copyright and moral rights for the publications made accessible in the public portal are retained by the authors and/or other copyright owners and it is a condition of accessing publications that users recognise and abide by the legal requirements associated with these rights.

- Users may download and print one copy of any publication from the public portal for the purpose of private study or research.

- You may not further distribute the material or use it for any profit-making activity or commercial gain

- You may freely distribute the URL identifying the publication in the public portal

If you believe that this document breaches copyright please contact us providing details, and we will remove access to the work immediately and investigate your claim 


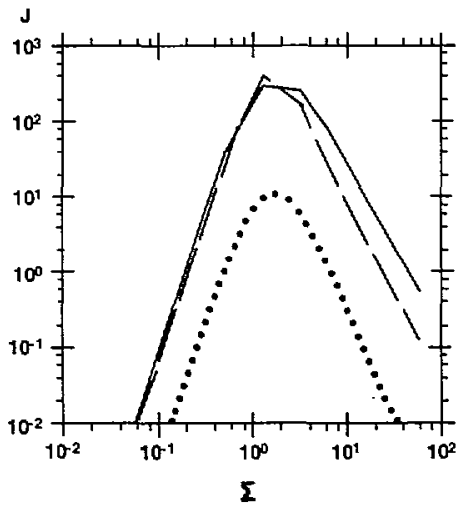

Fig. 7. Specular direction scattering, dependence on Rayleigh parameter.

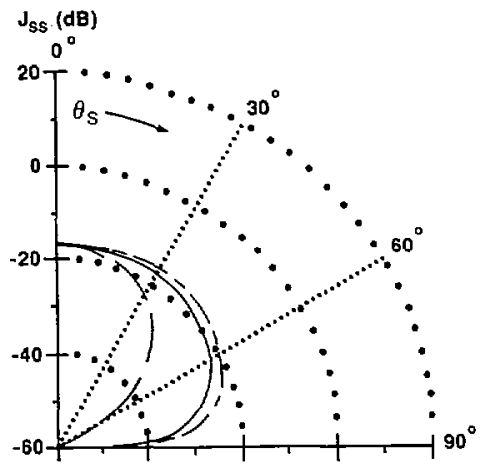

Fig. 8. Small scale $J_{S S}$ pattern, very rough surface with $\theta_{i}=85^{\circ}$.

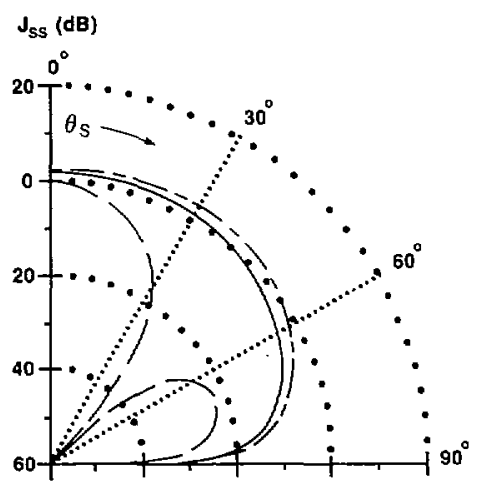

Fig. 9. Small scale $J_{S S}$ pattern, very rough surface with $\theta_{i}=45^{\circ}$.

apparent between the behavior for this type of surface and that for a surface with Gaussian height statistics. Thus, in the physical optics regime, the roughness dependence of the diffuse scattering angular distribution is insensitive to correlation function, height statistics, and angle of incidence.

Our result that the $J_{D}$ term always dominates the $J_{S}$ term for the physical optics regime $(T \gg \lambda)$ has implications for the discussions by Brown [6] concerning the significance of the specular incoherent scattering term $\left(J_{S}\right)$ for surfaces for which the decorrelated height statistics remain statistically dependent. The first point is that the criterion for using asymptotic diffuse scattering models [1], [2] in the case of exponential type surfaces (no statistical independence) is the same as would apply for statistically independent surfaces; that condition being negligible coherent power, not negligible incoherent specular scattering. The second point is that the nonexistence of such surfaces cannot be inferred from the lack of experimental observation of any such differences in scattering behavior.

The next set of findings concerns comparisons between the physical optics regime specular diffuse scattering behavior and that for backscatter conditions as described by Eom and Fung [5]. For purposes of comparison, inclusion of scattering matrix elements in our results just introduces a correlation invariant multiplier to the patterns of the slope related terms. For the specular diffuse scattering, the results for all rms height levels are sensitive to both surface slope and correlation function. The power law results are closer to the Gaussian correlation cases than those for the Bessel function correlation. Next, for both large and moderate $(\sim 1)$ Rayleigh parameter values, the diffuse specular scattering depends on the height statistics; it is only for small $\Sigma$ values that the diffuse scattering is insensitive to height distribution. Thus, the two basic differences from the backscatter trends are in the slope dependence for small $\Sigma$ and the sensitivity to height statistics at moderate $\Sigma$ values.

The results for the small height, perturbation theory regime, are more complicated. There is a definite dependence of the scattering patterns on incident angle as can be seen in (9). There is an explicit $\cos \theta_{s}$ term, in addition to implicit angle effects in the power spectra component from the Rayleigh parameter dependence. The power spectra terms have peaks in the specular direction for Gaussian and power law correlation, but a sharp null for the Bessel form. The combination of the two effects leads to distinctly different patterns as the incident angle changes. The relative insensitivity to correlation for $\theta_{i}=85^{\circ}$ arises from the dominance of the $\cos \theta_{s}$ term at that specular angle. For the perturbation theory regime, then, the diffuse scattering pattern is independent of height statistics and, except for magnitude, tends to be insensitive to roughness; however, it is strongly dependent on incident angle and correlation function.

\section{REFERENCES}

[1] P. Beckmann, and A. Spizzichino, The Scattering of Electromagnetic Waves from Rough Surfaces. New York: Macmillan, 1963.

[2] G. T. Ruck, D. E. Barrick, W. D. Stewart, and C. K. Krichbaum, Radar Cross Section Handbook, vol. 2. New York: Plenum, 1970.

[3] L. S. Miller, G. S. Brown, and G. S. Hayne, "Analysis of satellite altimeter signal characteristics and investigation of sea-truith data requirements," NASA-CR-137465, 1972.

[4] R. Cosgriff, W. Peake, and R. Taylor, Terrain Scattering Properties For Sensor System Design (Terrain Handbook II), Ohio State Univ., EES Bulletin 181, 1960.

[5] J. H. Eom, and A. K. Fung, "A comparison between backscattering coefficients using Gaussian and non-Gaussian surface statistics," IEEE Trans. Antennas Propagat., vol. AP-31, pp.635-638, July 1983.

[6] G. S. Brown, "Scattering from a class of randomly rough surfaces," Radio Sci., vol. 17, no. 5, pp. 1274-1280, 1982.

\section{Comments on Field Equivalence Principles}

JØRGEN APPEL-HANSEN, MEMBER, IEEE

Abstract-It is pointed out that often-used arguments based on a shortcircuit concept in presentations of field equivalence principles are not correct. An alternative presentation based on the uniqueness theorem is

Manuscript received January 8, 1986; revised February 7, 1986.

The author is with the Electromagnetics Institute, The Technical University of Denmark, DK-2800 Lyngby, Denmark.

IEEE Log Number 8612264 . 
given. It does not contradict the results obtained by using the short-circuit concept but provides further insight.

\section{The Principle BaSEd on the Short-Circuit ConcePt}

In a field equivalence principle, the actual sources are replaced by equivalent sources which provide a field identical to that of the actual sources in a certain region of space. The advantage of equivalence principles is that they may suggest approximate methods for evaluating the field and they may facilitate the understanding of electromagnetic problems.

Let the actual sources be placed inside a volume $V$ enclosed by a surface $S$. Furthermore, let us be interested in the electric field intensity $\bar{E}_{1}$ and magnetic field intensity $\bar{H}_{1}$ from the actual sources in the region outside $V$. The entire space is supposed to be homogeneous. It is assumed that the conditions for uniqueness of the solution are satisfied, i.e., there are no problems at cavity resonances, for example, when the region outside $V$ is an interior region.

In one of the equivalence principles, the original field $\bar{E}_{1}, \bar{H}_{1}$ is retained outside $V$ [1]-[3]. However, inside $V$ the actual sources are removed and the field is specified to be zero as illustrated in Fig. 1. According to the boundary conditions this specification requires an electric surface current $\bar{J}_{s}$ and a magnetic surface current $\bar{J}_{m s}$ on the surface $S$ given by

$$
\begin{gathered}
\bar{J}_{s}=\hat{n} \times \bar{H}_{1} \\
\bar{J}_{m s}=-\hat{n} \times \bar{E}_{1}
\end{gathered}
$$

where $\hat{n}$ is a unit vector of $S$ directed out of $V$ into the region with the field $\bar{E}_{1}, \bar{H}_{1}$. Since the field inside $V$ is specified, $\bar{J}_{s}$ and $\bar{J}_{m s}$ are referred to as specified currents. According to the uniqueness theorem the field outside $V$ is completely determined from either the tangential component of $\bar{E}_{1}$ or $\bar{H}_{1}$ on $S$. Various procedures can now be followed to find $\bar{E}_{1}, \bar{H}_{1}$.

One procedure is based on the following arguments. Let $\bar{J}_{s}$ and $\bar{J}_{m s}$ be chosen as impressed currents. Furthermore, since the field inside $V$ is zero, let $V$ be filled with a perfect electric conductor. This will short-circuit $\bar{J}_{s}$. The field can now be found from $\bar{J}_{m s}$ which radiates in the presence of the conductor. In some derivations it is stated that the surface of the conductor is "just behind" $S$ or the currents are "placed an infinitesimal distance in front of " $S$ " [2], [1]. In such derivations $\bar{J}_{s}$ cannot be "short-circuited" because there is an infinitesimal gap between $\bar{J}_{s}$ and the conductor. Therefore, such presentations are not correct. The purpose of this communication is to give a presentation that avoids the "short-circuit" and the gap concepts. It is hoped that the alternative derivation increases insight.

\section{The Principle Based on the Uniqueness Theorem}

Let the volume $V$ be filled with a conductor with infinite electric conductivity $\sigma$ and zero magnetic conductivity $\sigma_{m}$ as shown in Fig. 2 . This type of conductor in referred to as a perfect electric conductor. On the surface of this conductor an impressed magnetic surface current $\bar{J}_{m s}$ is placed. This current is chosen equal to the current specified by (2).

The current $\bar{J}_{m s}$ will induce no magnetic surface current because $\sigma_{m}$ $=0$. However, an electric surface current $\bar{J}_{s 2}$ may be induced on the surface $S$. Let the field outside $V$ as a result of $\bar{J}_{m s}$ and $\bar{J}_{s 2}$ be $\bar{E}_{2}, \bar{H}_{2}$. Due to $\sigma=\infty$, the field inside the perfect conductor is zero. Then, the boundary conditions require

$$
\begin{gathered}
\bar{J}_{s 2}=\hat{n} \times \bar{H}_{2} \\
\bar{J}_{m s}=-\hat{n} \times \bar{E}_{2} .
\end{gathered}
$$

Comparison of (2) and (4) shows that the tangential component of $\bar{E}_{2}$

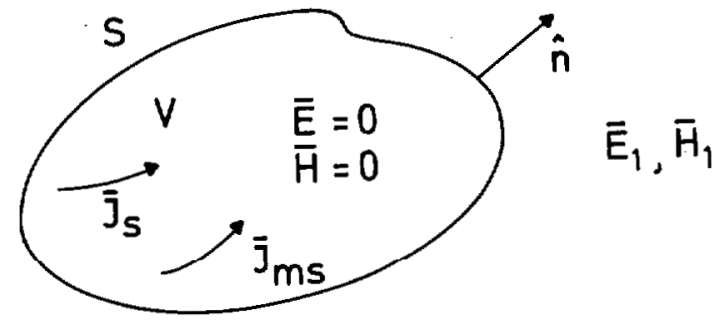

Fig. 1. Equivalence principle.

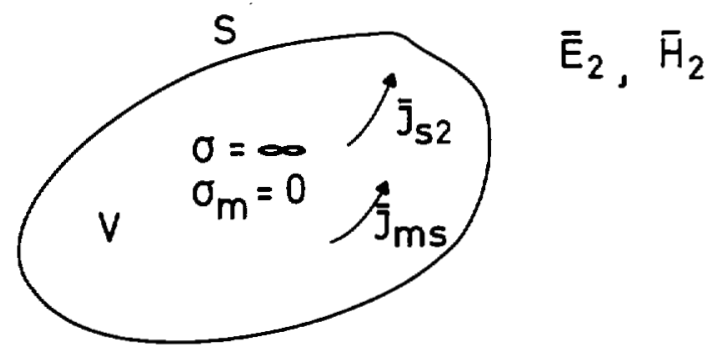

Fig. 2. Perfect electric conductor inside $V$.

is equal to the tangential component of $\bar{E}_{1}$. Then according to the uniqueness theorem $\bar{E}_{1}, \bar{H}_{1}$ and $\bar{E}_{2}, \bar{H}_{2}$ are identical. This means that

$$
\bar{J}_{s 2}=\bar{J}_{s} \text {. }
$$

Thus, in Fig. 2 the induced current $\bar{J}_{s 2}$ is equal to the current $\bar{J}_{s}$ specified in Fig. 1. It should be noted that in case $V$ was filled with a perfect magnetic conductor, $J_{s}$ would be chosen to be an impressed current and $\bar{J}_{m s}$ would then be induced. Thus, independent of the type of conductor the values of the currents are the same. It is only the manners in which the currents are obtained which differ, i.e., the perfect electric and magnetic conductors, provide two different examples of obtaining the currents specified in Fig. 1. To the author's knowledge this insight in the positions, the values and the types of currents does not appear from previous treatments of equivalence principles, some of which are cited in [1] and [3]. However, it should be noted that this insight is only obtained for the special conductors chosen above. In [2] it is mentioned that the total field must remain unchanged when matter is placed within $S$ and impressed currents are chosen equal to the specified currents. For this case it is in general difficult to find the induced currents.

It is interesting to note that on the surface $S$ of the perfect electric conductor in Fig. 2, a tangential electric field component exists on the free space side. This is due to the impressed $\bar{J}_{m s}$. It is probably the often-used requirement of zero tangential electric field which causes the introduction of the infinitesimal gap between the currents and the perfect conductor in the references cited above. See also the discussion in relation to Fig. 3. However, due to the introduction of the concepts $\bar{J}_{m s}$ and $\sigma_{m}$ in Maxwell's equations, a perfect electric conductor no longer requires (when a $\bar{J}_{m s}$ is impressed on the surface) that the tangential electric field is zero on its surface. This fact is seen from the boundary conditions taking into account that $\bar{J}_{m s}$ coincide with the surface. Therefore, when $V$ is filled with a perfect electric conductor and $\bar{J}_{m s}$ is impressed on its surface, it is not correct to state that the radiated field "must be determined so that $\hat{n} \times \bar{E}$ will vanish on the conducting surface" which is done in [3]. This is understood by noting that in the original problem $\hat{n} \times \bar{E}=\hat{n} \times \bar{E}_{1}$ does not vanish. However, as demonstrated below, the correct solution may be found in a limiting process during which use is made of the fact that $\hat{n}$ $\times \bar{E}$ vanishes as long as there is no magnetic surface current on the conductor. 


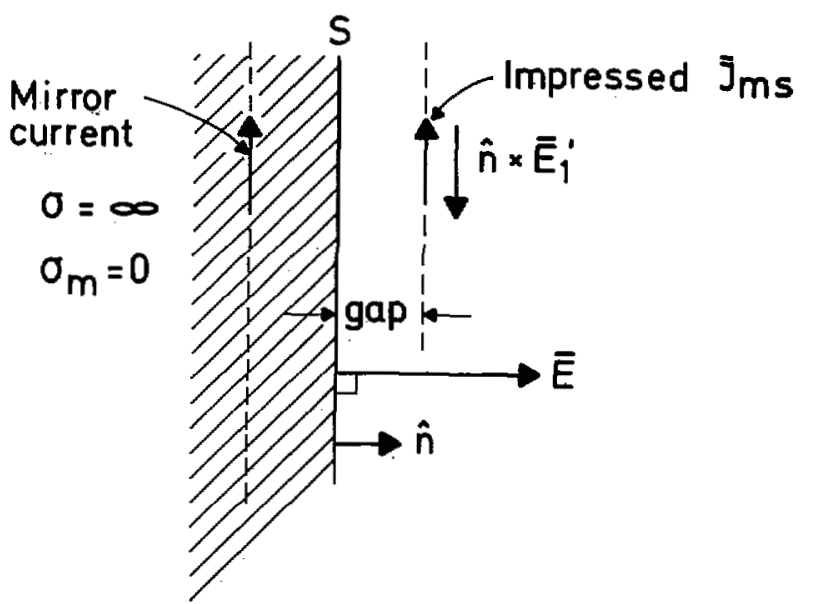

Fig. 3. Image theory.

The field $\bar{E}_{1}, \bar{H}_{1}$ (equal to $\bar{E}_{2}, \bar{H}_{2}$ ) may be calculated using image theory. This is understood by imagining $\widetilde{J}_{m s}$ at a distance from $S$. In case $S$ is an infinite plane the mirror current takes the same value as $\bar{J}_{m s}$. This is found by making use of a vanishing $\hat{n} \times \bar{E}$ on $S$. Then, in the limit of zero distance, $2 \bar{J}_{m s}$ provides the field $\bar{E}_{1}, \bar{H}_{1}$ outside $\boldsymbol{V}$. Inside $V, 2 \bar{J}_{m s}$ does not give zero field. Apparently the mirror current of $\bar{J}_{m s}$ is a magnetic surface current. This is not due to induced magnetic currents but due to the induced electric surface currents equal to $\bar{J}_{s}$ as discussed above. As long as there is a gap the induced currents keep the tangential electric field zero. In the limit of no gap there is a tangential component due to $\dot{J}_{m s}$ coinciding with $S$, i.e., the electric field changes discontinuously from having a zero tangential component as long as there is a gap to having a tangential component as the gap disappears. This is illustrated in Fig. 3. As the gap tends to zero the tangential electric field $\hat{n} \times E_{\mathrm{i}}^{\prime}$ in front of $\bar{J}_{m s}$ tends to $\hat{n} \times \bar{E}_{\text {l }}$.

Using image theory, it is also understood that impressed electric and magnetic currents give fields which tend to zero as the currents approach a perfect electric and magnetic conductor, respectively. Therefore, on perfect electric and magnetic conductors impressed electric and magnetic currents, respectively, cannot exist. Physically it may be said that the impressed currents are cancelled by equal and oppositely directed induced currents. In equivalent circuit terminology, the impressed currents are short-circuited. It should be noted that this requires that the impressed currents be tangential to the surface of the conductors. In the references cited above image theory is used neglecting the infinitesimal gap. Therefore, the same results are obtained in the two approaches of derivation.

\section{ACKNOWLEDGMENT}

The author wishes to acknowledge Jesper E. Hansen for commenting on the manuscript and helpful discussions.

\section{REFERENCES}

[1] R. E. Collin and J. F. Zucher, Antenna Theory, vol. 1. New York: McGraw-Hill, 1969, pp. 68-71.

[2] R. F. Harrington, Time-Harmonic Electromagnetic Fields. New York: McGraw-Hill, 1961, pp. 106-110.

[3] R. E. Collin, Antennas and Radiowave Propagation. New York: McGraw-Hill, 1985, p. 182.

\section{Correction to "Diffraction by a Half-Plane with Two Face Impedance Uniform Asymptotic Expansion for Plane Wave and Arbitrary Line Source Incidence"}

\author{
SUBRATA SANYAL, MEMBER, IEEE, AND ASOKE K. BHATTACHARYYA, \\ SENIOR MEMBER, IEEE
}

In (10) on page 721 of the above communication ${ }^{i}$ it was stated that the diffracted field is given by (6f). This is not correct. Instead, it is expressed as

$$
u^{d}(\rho, \phi) \sim-u_{0}^{i} D\left(\phi, \phi_{0}\right) \exp (j k \rho) / \sqrt{\rho}
$$

where $u_{0}^{i}$ is the value of the incident field at the edge and $D\left(\phi, \phi_{0}\right)$ is given by [10, eq. (60)] in our communication. The diffracted field expansion takes into account the surface wave fields in Rawlin's work unlike what we had stated earlier (page 721, second column, second paragraph). The error is regretted.

Manuscript received August 13, 1986.

S. Sanyal is with the Radar and Communication Centre, Indian Institute of Technology, Kharagpur 721302, India.

A. K. Bhattacharyya is with the Department of Electrical Engineering, Concordia University, 7141 Sherbrooke St. West, Montreal, Quebec H4B1R6, Canada, on leave from the Radar and Communication Centre, Indian Institute of Technology, Kharagpur 721302, India.

IEEE Log Number 8612251.

'S. Sanyal and A. K. Bhattacharyya, IEEE Trans. Antennas Propagat., vol. AP-34, no. 5, pp. 718-723, May 1986. 